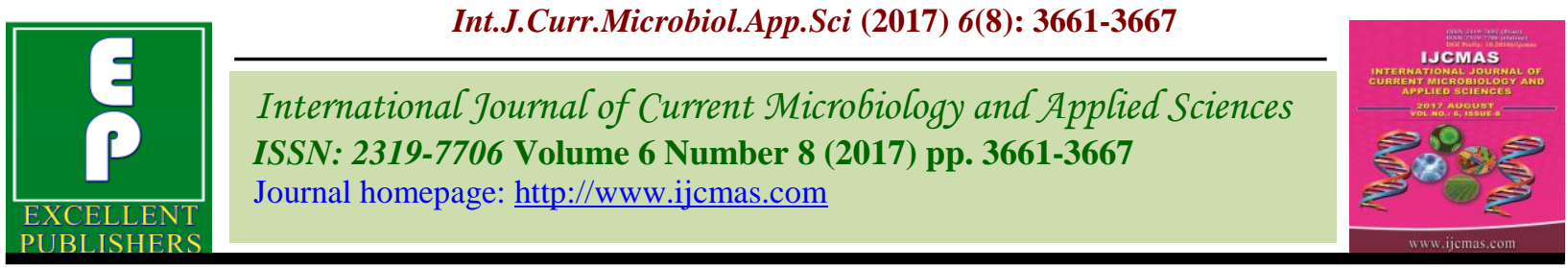

Original Research Article

https://doi.org/10.20546/ijcmas.2017.608.443

\title{
Field Efficacy of Seed Dressing Fungicides against Seed Borne Diseases of Cotton
}

\author{
S.L. Bhattiprolu* \\ Acharya N G Ranga Agricultural University, Regional Agricultural Research Station, \\ Lam, Guntur - 522 034, Andhra Pradesh, India \\ *Corresponding author
}

A B S T R A C T

Seed dressing fungicides viz., thiram, and carboxin and combination product of thiram + carboxin were evaluated in cotton during kharif seasons of 2012 to 2014. Cotton variety

Keywords Narasimha was raised in plots of 31.5 sq. m adopting a spacing of 105 x $60 \mathrm{~cm}$. Ten treatments viz., T1 - Seed treatment (ST) with thiram @ 2g/kg seed; T2 - ST with thiram

Cotton, Seed borne diseases, Seed dressing fungicides. @ 3g/kg seed; T4 - ST with thiram @ 4g/kg seed; T4 - ST with carboxin @ 1g/kg seed; T5 - ST with carboxin@2g/kg seed; T6 - ST with carboxin@3g/kg seed; T7 - ST with thiram + carboxin@ 2.5g/kg seed; T8-ST with thiram + carboxin @ 3.5g/kg seed; T9-ST with thiram + carboxin @ 3.5g/kg seed and T10 - Untreated control were imposed at the time of sowing in randomized block design with three replications. Data on seed borne diseases was collected by adopting $0-4$ scale and disease intensity was calculated. Treatment wise yield data was recorded and Incremental Benefit Cost Ratio (IBCR) was calculated. Combination of carboxin $37.5 \% \mathrm{WP}+$ thiram $37.5 \%$ DS $4.5 \mathrm{~g} / \mathrm{kg}$ resulted in lowest mortality $(4.11 \%)$ and lowest intensity of Alternaria leaf spot $(4.11 \%)$ with maximum yield of $9.65 \mathrm{q} / \mathrm{ha}$. IBCR of different treatments varied between 4.82 and 4.93 . Hence thiram @3g/kg or carboxin @ 2g/kg or combination product @ 3.5 $/ \mathrm{kg}$ is recommended for seed treatment in cotton.

\section{Introduction}

Cotton is an important commercial crop in India with a production of 351 lakh bales of $170 \mathrm{~kg}$ lint in 2016-2017 from an area of 105 lakh ha with a productivity of $568 \mathrm{~kg} / \mathrm{ha}$, which is far behind the leading countries. Andhra Pradesh stood $6^{\text {th }}$ in area (4.49 lakh ha) but $8^{\text {th }}$ in production (13.10 lakh bales) and $2^{\text {nd }}$ in productivity $(719 \mathrm{~kg} / \mathrm{ha})$ during 2016 - 2017 (Anonymous, 2017). Seed borne diseases affect germination and cause seedling mortality. Several pathogens including bacterial blight (Xanthomonas axonopodis pv malvacearum), fungal leaf spots caused by Alternaria macrospora, A. alternata, Myrothecium roridum,

Colletotrichum capsici are seed borne while Rhizoctonia solani, $R$. bataticola (Macrophomina bataticola), Fusarium oxysposum f. sp. Vasinfectum, Verticillium dahlia are mainly soil borne in cotton.

In Andhra Pradesh Alternaria leaf spot is the most commonly occurring disease starting from the germination and cause economic losses under congenial conditions (Bhattiprolu and Prasada Rao, 2009).

Importance of seed treatments in sustainable agriculture was reviewed by Sharma et al., (2015). Seed treatment with thiram @ 3g or 
carboxin @ 2g or captan @ 3g or carbendazim @ 2g was recommended to manage seed/soil borne diseases in cotton. However these seed dressing fungicides need validation in course of time. Hence the present investigation was carried out to verify the efficacy of label claimed fungicides against seed and/or soil borne diseases in cotton.

\section{Materials and Methods}

A field experiment was conducted at Regional Agricultural Research Station, Lam, Guntur during kharif seasons of 2012 to 2014 to validate the seed dressing fungicides against seed borne diseases of cotton.

The label claimed chemicals namely thiram, carboxin and combination product of thiram + carboxin were tested at three doses each. Cotton variety Narasimha was raised in plots of 31.5 sq. m adopting a spacing of 105 x 60 $\mathrm{cm}$. Ten treatments viz., T1 - Seed treatment (ST) with thiram @ 2g/kg seed; T2 - ST with thiram@3g/kg seed; T4 - ST with thiram@ 4g/kg seed; T4 - ST with carboxin @ 1g/kg seed; T5 - ST with carboxin @ 2g/kg seed; T6 - ST with carboxin @ 3g/kg seed; T7 - ST with thiram + carboxin @ 2.5g/kg seed; T8 ST with thiram + carboxin @ 3.5g/kg seed; T9 - ST with thiram + carboxin @ $3.5 \mathrm{~g} / \mathrm{kg}$ seed and T10 - Untreated control were imposed at the time of sowing in randomized block design with three replications. Data on germination and plant stand was recorded and seedling mortality was calculated 30days after sowing.

Data on seed borne diseases was collected up to 45days of sowing by adopting 0-4scale (Sheo Raj, 1988): $0=$ No disease; $1=<5 \% ; 2$ $=6-20 \% ; 3=20-40 \%$ and $4=>40 \%$ leaf area is diseased. Depending on the scores collected percent disease intensity (PDI) was calculated using the formula of Wheeler (1969).

\author{
Sum of all the numerical ratings

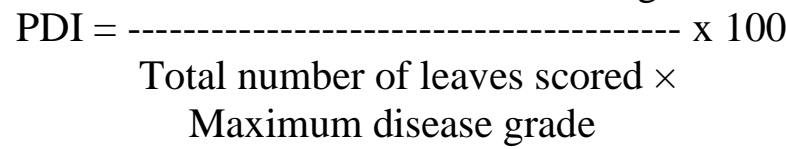

Percent disease control in each treatment was calculated. Recommended protection measures against insect pests were taken on need basis. Yield data from three replications of each treatment was recorded. Decrease / increase in the disease/ yield over control were calculated using the formula:

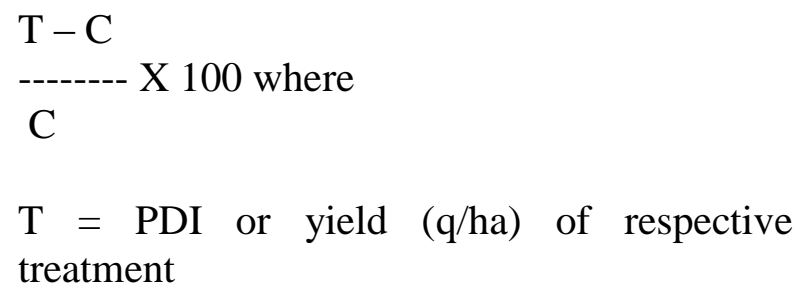

Treatment wise Incremental Benefit Cost Ratio (IBCR) was calculated by dividing additional benefit with additional cost.

\section{Results and Discussion}

During 2012-13 and 2014-15 carboxin 37.5\% $\mathrm{WP}+$ thiram $37.5 \%$ DS $4.5 \mathrm{~g} / \mathrm{kg}$ recorded the lowest mortality of 6.0 and 3.5, respectively while thiram 75WS @ 4g/kg, carboxin 75\% WP @ $3 \mathrm{~g} / \mathrm{kg}$ and carboxin 37.5\% WP + thiram $37.5 \%$ DS $3.5 \mathrm{~g} / \mathrm{kg}$ were at par with it. Carboxin $37.5 \% \mathrm{WP}+$ thiram $37.5 \% \mathrm{DS}$ $4.5 \mathrm{~g} / \mathrm{kg}$ (1.67\%), carboxin 75\% WP @ 3g/kg $(1.67 \%)$ and thiram 75WS @ 4g/kg (2.5\%) were on par and statistically superior to other treatments during 2013-14 (Table 1). Pooled data (2012-14) revealed that carboxin $37.5 \%$ $\mathrm{WP}+$ thiram $37.5 \%$ DS $4.5 \mathrm{~g} / \mathrm{kg}$ resulted in the lowest seedling mortality (4.0\%); carboxin 75\% WP @ 3g/kg (4.11\%) and thiram 75WS @ 4g/kg (4.61\%) were on par with it while control plots recorded $13.27 \%$. Tomer et al., (2012) obtained $63.96 \%$ to 
$68.77 \%$ germination by protecting against seed borne Myrothecium roridum, Aspergillus niger and Curvularia lunata in $\mathrm{J} 4$ cotton cultivar.

Alternaria leaf spot was observed on seedlings (Fig.1 a) and pathogen was isolated and confirmed for pathogenicity (Fig.1b). Carboxin 37.5\% WP + thiram 37.5\% DS $4.5 \mathrm{~g} / \mathrm{kg}$ recorded the lowest intensity of Alternaria leaf spot $(2.5 \%)$ whereas carboxin 75\% WP @ 3g/kg (2.83\%), carboxin 37.5\% $\mathrm{WP}+$ thiram $37.5 \%$ DS $3.5 \mathrm{~g} / \mathrm{kg}(3.33 \%)$ and thiram 75WS @ 4g/kg (4\%) were statistically at par during 2012-13 (Table 1). All the treatments were superior to untreated control during 2013-14. Carboxin 37.5\% WP + thiram $37.5 \%$ DS $4.5 \mathrm{~g} / \mathrm{kg}$ recorded the lowest intensity of Alternaria leaf spot (3.67\%) whereas carboxin 75\% WP @ 3g/kg (4.33\%), carboxin $37.5 \% \mathrm{WP}+$ thiram $37.5 \%$ DS $3.5 \mathrm{~g} / \mathrm{kg}$ (4.33), thiram 75WS @ 4g/kg (4.67\%) and carboxin 75\% WP @ 2g/kg (5.0) were statistically at par during 2014-15. Pooled results revealed that combination of carboxin $37.5 \%$ WP + thiram $37.5 \%$ DS $4.5 \mathrm{~g} / \mathrm{kg}$ resulted in lowest intensity of Alternaria leaf spot $(4.11 \%)$ while the same at $3.5 \mathrm{~g} / \mathrm{kg}(5.22 \%)$ or carboxin @ 3g/kg (4.61\%) were found on par with it while control plots recorded disease intensity of $15.14 \%$.
Maximum reduction of $72.85 \%$ was obtained in carboxin $37.5 \% \mathrm{WP}+$ thiram $37.5 \% \mathrm{DS}$ $4.5 \mathrm{~g} / \mathrm{kg}$ followed by $69.55 \%$ in carboxin $75 \%$ WP @ 3g/kg and 65.52\% in carboxin 37.5\% $\mathrm{WP}+$ thiram $37.5 \%$ DS $3.5 \mathrm{~g} / \mathrm{kg}$ (Table 1). These results were in conformity with previous reports. Tomer et al., (2012) evaluated carboxin @ 2g/kg and found highly effective in controlling the seed borne pathogens. Ebadollah Baniani et al., (2015) recommended Goucho and carboxin-thiram, Larvin and carboxin-thiram for seed disinfection cotton. Oktay Erdogan et al., (2016) found that vitavax was very effective against Rhizoctonia solani and Fusarium sp. with highest germination $(76.1 \%)$ in cotton cultivar Carman. Eisa (Nawal) et al., (2005) recorded vitavax-T 70 (carboxin + thiram $70 \%$ ) and Vitavax-T40 (carboxin + thiram $40 \%$ ) were the best seed treatments root pathogens in cotton cultivars Giza-86 and Giza-89 with increased yields. Asghar Heydari (2015) observed that delinting with 98\% acid was more effective against Rhizoctonia solani causing seed decay (rot) and seedling damping-off in Sahel and Varamin cotton varieties in Iran. Seed delinting with 80 and $60 \%$ acid and seed treatment with carboxin-thiram fungicide also significantly reduced the disease incidence in comparison with linted seeds.

Fig.1a Seed borne infection by Alternaria macrospora in cotton;

1b Culture of Alternaria macrospora

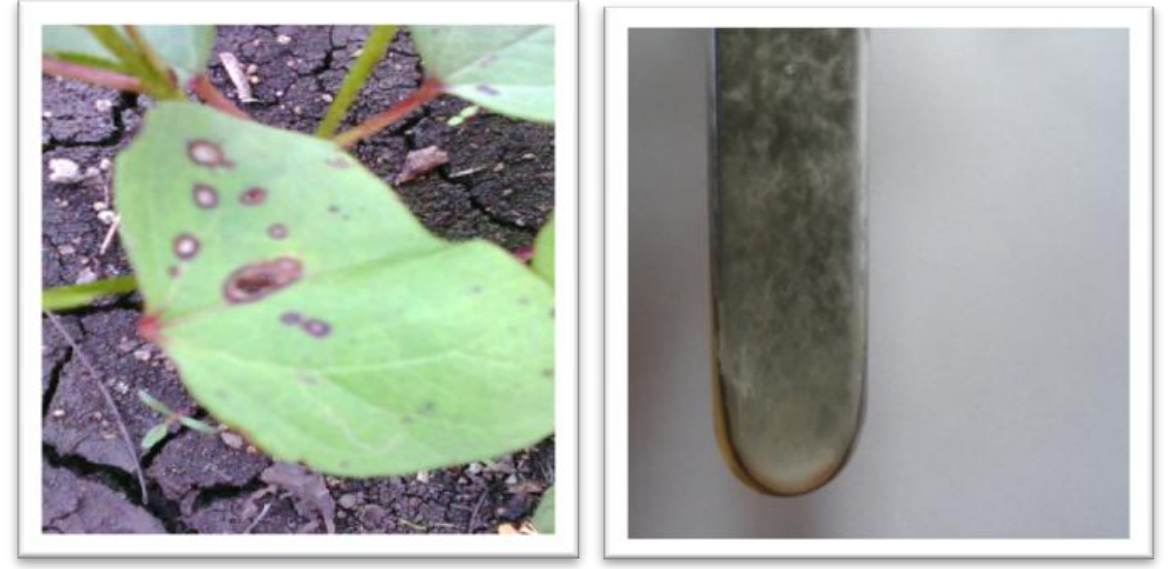


Table.1 Efficacy of seed dressing chemicals on seed and soil borne diseases of cotton (Pooled data, 2012-2014)

\begin{tabular}{|c|c|c|c|c|c|c|c|c|c|c|}
\hline \multirow[t]{2}{*}{$\begin{array}{l}\text { T. } \\
\text { No. }\end{array}$} & \multirow[t]{2}{*}{ Treatment } & \multicolumn{3}{|c|}{ Seed ling Mortality (\%) } & \multirow[t]{2}{*}{ Mean } & \multicolumn{3}{|c|}{$\begin{array}{l}\text { Intensity of Alternaria leaf spot } \\
(\%)\end{array}$} & \multirow[t]{2}{*}{ Mean } & \multirow{2}{*}{$\begin{array}{l}\text { Decrease in } \\
\text { Alternaria leaf spot } \\
(\%)\end{array}$} \\
\hline & & $2012-13$ & 2013-14 & 2014-15 & & $2012-13$ & 2013-14 & 2014-15 & & \\
\hline 1 & Thiram75WS @ 2g/kg & $18.00^{\mathrm{e}}$ & $8.33^{\mathrm{de}}$ & $7.50^{\mathrm{h}}$ & $12.89^{\mathrm{de}}$ & $\begin{array}{l}4.96^{\text {defg }} \\
(12.92)\end{array}$ & $\begin{array}{l}9.00^{\mathrm{a}} \\
(17.45)\end{array}$ & $\begin{array}{l}7.67^{\mathrm{f}} \\
(16.06)\end{array}$ & $\begin{array}{l}7.15^{\mathrm{de}} \\
(15.51)\end{array}$ & 52.77 \\
\hline 2 & Thiram75WS @ 3g/kg & $12.67^{\mathrm{cd}}$ & $5.83^{b c}$ & $5.33^{\text {cdef }}$ & $08.28^{\mathrm{bc}}$ & $\begin{array}{l}4.83^{\text {cdefg }} \\
(12.66)\end{array}$ & $\begin{array}{l}8.33^{\mathrm{a}} \\
(16.74)\end{array}$ & $\begin{array}{l}6.33^{\mathrm{def}} \\
(14.54)\end{array}$ & $\begin{array}{l}5.89^{\mathrm{cd}} \\
(14.0)\end{array}$ & 61.10 \\
\hline 3 & Thiram 75WS @ 4g/kg & $6.67^{\mathrm{ab}}$ & $2.50^{\mathrm{a}}$ & $4.00^{\mathrm{ab}}$ & $04.61^{\mathrm{a}}$ & $\begin{array}{l}4.00^{\mathrm{abcd}} \\
(11.54)\end{array}$ & $\begin{array}{l}7.67^{\mathrm{a}} \\
(16.56)\end{array}$ & $\begin{array}{l}4.67^{\text {abcd }} \\
(12.46)\end{array}$ & $\begin{array}{l}5.50^{\mathrm{bc}} \\
(13.56)\end{array}$ & 63.67 \\
\hline 4 & $\begin{array}{l}\text { Carboxin 75\% WP @ } \\
1 \mathrm{~g} / \mathrm{kg}\end{array}$ & $26.67^{f}$ & $9.17^{\mathrm{de}}$ & $5.83^{\text {cdefg }}$ & $13.39^{\mathrm{e}}$ & $\begin{array}{l}5.17^{\text {defg }} \\
(13.18)\end{array}$ & $\begin{array}{l}8.33^{\mathrm{a}} \\
(16.74)\end{array}$ & $\begin{array}{l}6.33^{\mathrm{def}} \\
(14.54)\end{array}$ & $\begin{array}{l}6.44^{\text {cde }} \\
(14.65)\end{array}$ & 57.46 \\
\hline 5 & $\begin{array}{l}\text { Carboxin 75\% WP @ } \\
2 \mathrm{~g} / \mathrm{kg}\end{array}$ & $20.0^{\mathrm{e}}$ & $5.83^{\mathrm{bc}}$ & $5.00^{\text {bcde }}$ & $10.28^{\mathrm{cd}}$ & $\begin{array}{l}4.50^{\text {cdef }} \\
(12.25)\end{array}$ & $\begin{array}{l}7.67^{\mathrm{a}} \\
(16.56)\end{array}$ & $\begin{array}{l}5.00^{\text {abcde }} \\
(12.92)\end{array}$ & $\begin{array}{l}5.72^{\mathrm{bc}} \\
(13.81)\end{array}$ & 62.22 \\
\hline 6 & $\begin{array}{l}\text { Carboxin 75\% WP @ } \\
3 \mathrm{~g} / \mathrm{kg}\end{array}$ & $6.67^{\mathrm{ab}}$ & $1.67^{\mathrm{a}}$ & $4.67^{\mathrm{abc}}$ & $04.11^{\mathrm{a}}$ & $\begin{array}{l}2.83^{\mathrm{ab}} \\
(9.63) \\
\end{array}$ & $\begin{array}{l}6.67^{\mathrm{a}} \\
(14.95) \\
\end{array}$ & $\begin{array}{l}4.00^{\mathrm{ab}} \\
(11.54) \\
\end{array}$ & $\begin{array}{l}4.61^{\mathrm{ab}} \\
(12.39) \\
\end{array}$ & 69.55 \\
\hline 7 & $\begin{array}{l}\text { Carboxin 37.5\% WP + } \\
\text { Thiram } 37.5 \% \mathrm{DS} \\
2.5 \mathrm{~g} / \mathrm{kg}\end{array}$ & $10.67^{\mathrm{cd}}$ & $7.50^{\mathrm{cd}}$ & $5.67^{\text {cdefg }}$ & $07.95^{\mathrm{b}}$ & $\begin{array}{l}4.17^{\text {bcde }} \\
(11.33)\end{array}$ & $\begin{array}{l}7.67^{\mathrm{a}} \\
(16.56)\end{array}$ & $\begin{array}{l}5.67^{\text {bcd }} \\
(13.75)\end{array}$ & $\begin{array}{l}5.56^{\mathrm{bc}} \\
(13.63)\end{array}$ & 63.28 \\
\hline 8 & $\begin{array}{l}\text { Carboxin 37.5\% WP + } \\
\text { Thiram } 37.5 \% \text { DS } \\
3.5 \mathrm{~g} / \mathrm{kg}\end{array}$ & $9.34^{\mathrm{abc}}$ & $5.00^{b}$ & $4.83^{\mathrm{bcd}}$ & $06.22^{\mathrm{ab}}$ & $\begin{array}{l}3.33^{\mathrm{abc}} \\
(10.47)\end{array}$ & $\begin{array}{l}6.67^{\mathrm{a}} \\
(14.95)\end{array}$ & $\begin{array}{l}4.33^{a b c} \\
(11.97)\end{array}$ & $\begin{array}{l}5.22^{\mathrm{abc}} \\
(13.18)\end{array}$ & 65.52 \\
\hline 9 & $\begin{array}{l}\text { Carboxin 37.5\% WP + } \\
\text { Thiram } 37.5 \% \text { DS } \\
4.5 \mathrm{~g} / \mathrm{kg}\end{array}$ & $6.00^{\mathrm{a}}$ & $1.67^{\mathrm{a}}$ & $3.50^{\mathrm{a}}$ & $04.00^{\mathrm{a}}$ & $\begin{array}{l}2.5^{\mathrm{a}} \\
(9.10)\end{array}$ & $\begin{array}{l}6.00^{\mathrm{a}} \\
(14.19)\end{array}$ & $\begin{array}{l}3.67^{\mathrm{a}} \\
(11.02)\end{array}$ & $\begin{array}{l}4.11^{\mathrm{a}} \\
(11.68)\end{array}$ & 72.85 \\
\hline 10 & Untreated control & $17.34^{\mathrm{e}}$ & $15.83^{\mathrm{f}}$ & $9.17^{\mathrm{i}}$ & $16.17^{f}$ & $\begin{array}{l}13.27^{\mathrm{h}} \\
(21.39)\end{array}$ & $\begin{array}{l}23.0^{\mathrm{b}} \\
(28.56)\end{array}$ & $\begin{array}{l}15.33^{\mathrm{g}} \\
(23.03)\end{array}$ & $\begin{array}{l}15.14^{\mathrm{f}} \\
(22.79) \\
\end{array}$ & \\
\hline & $\mathrm{CD}(\mathrm{p}=0.05)$ & 3.87 & 2.12 & 1.29 & 2.67 & 1.61 & 3.05 & 1.98 & 1.25 & \\
\hline & CV (\%) & 17.1 & 19.5 & 12.6 & 17.7 & 18.9 & 19.5 & 18.3 & 11.1 & \\
\hline
\end{tabular}

*Figures in parentheses are transformed values. Figures marked with same letters are not significantly different 
Table.2 Efficacy of seed dressing chemicals on seed and soil borne diseases of cotton (Pooled data, 2012-2014)

\begin{tabular}{|c|c|c|c|c|c|c|}
\hline \multirow[t]{2}{*}{ T. No. } & \multirow[t]{2}{*}{ Treatment } & \multicolumn{3}{|c|}{ Seed Cotton Yield (q/ha) } & \multirow{2}{*}{$\begin{array}{l}\text { Mean } \\
\text { Yield (q/ha) }\end{array}$} & \multirow{2}{*}{$\begin{array}{l}\text { Increase in } \\
\text { yield }(\%)\end{array}$} \\
\hline & & $2012-13$ & 2013-14 & $2014-15$ & & \\
\hline 1 & Thiram 75WS @2g/kg & 6.57 & $7.10^{\mathrm{ef}}$ & $10.11^{\mathrm{bc}}$ & $7.82^{\mathrm{d}}$ & 11.87 \\
\hline 2 & Thiram 75WS @3g/kg & 7.10 & $7.55^{\text {cdef }}$ & $10.27^{\mathrm{bc}}$ & $8.31^{\mathrm{c}}$ & 18.28 \\
\hline 3 & Thiram 75WS@4g/kg & 7.26 & $8.15^{\text {abcde }}$ & $11.85^{\mathrm{a}}$ & $9.09^{\mathrm{a}}$ & 30.04 \\
\hline 4 & Carboxin 75\% WP @1g/kg & 6.67 & $7.30^{\mathrm{def}}$ & $10.16^{\mathrm{b}}$ & $8.04^{b}$ & 15.02 \\
\hline 5 & Carboxin 75\% WP @ 2g/kg & 6.97 & $8.45^{\text {abcd }}$ & $12.33^{\mathrm{a}}$ & $9.25^{\mathrm{ab}}$ & 32.33 \\
\hline 6 & Carboxin 75\% WP @ 3g/kg & 7.23 & $8.82^{\mathrm{ab}}$ & $12.42^{\mathrm{a}}$ & $9.49^{\mathrm{a}}$ & 35.77 \\
\hline 7 & Carboxin $37.5 \% \mathrm{WP}+$ Thiram $37.5 \%$ DS $2.5 \mathrm{~g} / \mathrm{kg}$ & 6.87 & $7.77^{\text {cde }}$ & $10.42^{\mathrm{b}}$ & $8.35^{\mathrm{c}}$ & 19.46 \\
\hline 8 & Carboxin $37.5 \% \mathrm{WP}+$ Thiram $37.5 \%$ DS $3.5 \mathrm{~g} / \mathrm{kg}$ & 7.33 & $8.65^{\mathrm{abc}}$ & $12.60^{\mathrm{a}}$ & $9.53^{\mathrm{a}}$ & 34.34 \\
\hline 9 & Carboxin $37.5 \% \mathrm{WP}+$ Thiram $37.5 \%$ DS $4.5 \mathrm{~g} / \mathrm{kg}$ & 7.55 & $9.24^{\mathrm{a}}$ & $12.88^{\mathrm{a}}$ & $9.65^{\mathrm{a}}$ & 38.05 \\
\hline \multirow[t]{3}{*}{10} & Untreated control & 6.01 & $6.49^{f}$ & $8.45^{\mathrm{c}}$ & $6.99^{d}$ & \\
\hline & $\mathrm{CD}$ at $5 \%$ & NS & 1.25 & 1.21 & 1.04 & \\
\hline & $\mathrm{CV}(\%)$ & 14.8 & 9.2 & 6.3 & 7.0 & \\
\hline
\end{tabular}

*Figures in parentheses are transformed values. Figures marked with same letters are not significantly different

Table.3 Economics of treatments against seed and soil borne diseases of cotton (Pooled data, 2012-2014)

\begin{tabular}{|c|c|c|c|c|c|c|c|c|c|c|c|}
\hline \multirow[t]{2}{*}{ T. No. } & \multirow[t]{2}{*}{ Treatment } & \multicolumn{3}{|c|}{ Additional Expenditure (Rs) } & \multicolumn{3}{|c|}{ Additional Returns (Rs) } & \multicolumn{3}{|c|}{ Incremental Benefit Cost Ratio (IBCR) } & \multirow{2}{*}{$\begin{array}{l}\text { Mean } \\
\text { IBCR }\end{array}$} \\
\hline & & $2013-14$ & $2013-14$ & $2014-15$ & $2013-14$ & 2013-14 & $2014-15$ & 2013-14 & 2013-14 & $2014-15$ & \\
\hline 1 & Thiram 75WS @2g/kg & 453 & 493 & 1333 & 2224 & 2623 & 6059 & 4.91 & 5.32 & 4.55 & 4.93 \\
\hline 2 & Thiram 75WS @3g/kg & 879.5 & 855.5 & 1463.5 & 4251 & 4558 & 6643 & 4.83 & 5.33 & 4.54 & 4.90 \\
\hline 3 & Thiram 75WS @4g/kg & 1010 & 1338 & 2730 & 4875 & 7138 & 12410 & 4.83 & 5.33 & 4.55 & 4.90 \\
\hline 4 & $\begin{array}{l}\text { Carboxin 75\% WP @ } \\
1 \mathrm{~g} / \mathrm{kg}\end{array}$ & 535 & 655 & 1375 & 2574 & 3483 & 6241 & 4.81 & 5.32 & 4.54 & 4.89 \\
\hline 5 & $\begin{array}{l}\text { Carboxin 75\% WP @ } \\
2 \mathrm{~g} / \mathrm{kg}\end{array}$ & 782 & 1582 & 3118 & 3744 & 8428 & 14162 & 4.79 & 5.33 & 4.54 & 4.89 \\
\hline 6 & $\begin{array}{l}\text { Carboxin 75\% WP @ } \\
3 \mathrm{~g} / \mathrm{kg}\end{array}$ & 997 & 1885 & 3197 & 4758 & 10019 & 14490 & 4.77 & 5.32 & 4.53 & 4.87 \\
\hline 7 & $\begin{array}{l}\text { Carboxin } 37.5 \% \text { WP + } \\
\text { Thiram } 37.5 \% \text { DS } 2.5 \mathrm{~g} / \mathrm{kg}\end{array}$ & 712 & 1048 & 1600 & 3354 & 5504 & 7190 & 4.71 & 5.25 & 4.49 & 4.82 \\
\hline 8 & $\begin{array}{l}\text { Carboxin } 37.5 \% \text { WP + } \\
\text { Thiram } 37.5 \% \text { DS } 3.5 \mathrm{~g} / \mathrm{kg}\end{array}$ & 1089 & 1761 & 3353 & 5148 & 10098 & 14273 & 4.73 & 5.73 & 4.26 & 4.91 \\
\hline 9 & $\begin{array}{l}\text { Carboxin } 37.5 \% \text { WP + } \\
\text { Thiram } 37.5 \% \text { DS } 4.5 \mathrm{~g} / \mathrm{kg}\end{array}$ & 1275 & 2243 & 3587 & 6006 & 11825 & 16169 & 4.71 & 5.27 & 4.51 & 4.83 \\
\hline 10 & Untreated control & 0 & 0 & 0 & 0 & 0 & 0 & 0 & 0 & 0 & 0 \\
\hline
\end{tabular}


Carboxin + thiram @ 0.2\% was found most effective against seed borne fungal infections including A. alternata, Fusarium sp. and Xanthomonas axonopodis pv malvacearum by rolled towel method and controlled Alternaria blight in field (Hanasi, 2013).

Seed cotton yield was numerically superior to control in all the treatments during 2012-13 (Table 2). Carboxin 37.5\% WP + thiram $37.5 \% \mathrm{DS} 4.5 \mathrm{~g} / \mathrm{kg}$ recorded the highest yield of $9.24 \mathrm{q} / \mathrm{ha}$ while carboxin $75 \%$ WP @ $3 \mathrm{~g} / \mathrm{kg}$ $(8.82 \mathrm{q} / \mathrm{ha})$, carboxin $37.5 \% \mathrm{WP}+$ thiram $37.5 \%$ DS $3.5 \mathrm{~g} / \mathrm{kg}(8.65 \mathrm{q} / \mathrm{ha})$ and thiram $75 \mathrm{WS} @ 4 \mathrm{~g} / \mathrm{kg}(8.15 \mathrm{q} / \mathrm{ha})$ recorded on par yields during 2013-14. Carboxin 37.5\% WP + thiram $37.5 \%$ DS $4.5 \mathrm{~g} / \mathrm{kg}$ also recorded the highest yield of 12.88q/ha during 2014-15 followed by carboxin $37.5 \% \mathrm{WP}+$ thiram $37.5 \%$ DS $3.5 \mathrm{~g} / \mathrm{kg}$ (12.60q/ha), carboxin $75 \%$ WP @ 3g/kg (12.42q/ha), carboxin 75\% WP @ 2g/kg (12.33q/ha) and thiram 75WS @ $4 \mathrm{~g} / \mathrm{kg}(11.85 \mathrm{q} / \mathrm{ha})$. With respect to pooled data on seed cotton yield, carboxin $37.5 \% \mathrm{WP}$ + thiram $37.5 \%$ DS $4.5 \mathrm{~g} / \mathrm{kg}$ recorded maximum yield of $9.65 \mathrm{q} / \mathrm{ha}$ while the same at $3.5 \mathrm{~g} / \mathrm{kg} \quad(9.53 \mathrm{q} / \mathrm{ha})$, carboxin @ $3 \mathrm{~g} / \mathrm{kg}$ $(9.49 \mathrm{q} / \mathrm{ha})$ or carboxin @ 2g/kg $(9.25 \mathrm{q} / \mathrm{ha})$ and thiram @ 4g/kg (9.09q/ha) were found on par with it. Untreated control plot recorded 6.99q/ha.

Maximum yield increase of $38.05 \%$ was obtained with carboxin $37.5 \% \mathrm{WP}+$ thiram $37.5 \%$ DS $4.5 \mathrm{~g} / \mathrm{kg}$ followed by $35.77 \%$ with carboxin 75\% WP @ 3g/kg, carboxin 37.5\% $\mathrm{WP}+$ thiram $37.5 \%$ DS $3.5 \mathrm{~g} / \mathrm{kg}(34.34 \%)$, carboxin 75\% WP @ 2g/kg (32.33\%) and Thiram 75WS @ 4g/kg (30.04\%) (Table 2).

Incremental Benefit Cost Ratio (IBCR) of different treatments varied between 4.82 and 4.93 (Table 3). Based on these results thiram @ $3 \mathrm{~g} / \mathrm{kg}$ or carboxin @ $2 \mathrm{~g}$ or combination product@3.5g is recommended for seed treatment in cotton.

\section{Acknowledgements}

The author expresses gratitude to Indian Council of Agricultural Research (ICAR) for grant of fund for the Project work under All India Coordinated Cotton Improvement Project. Author also acknowledges the Associate Director of Research, RARS, Lam and Principal Scientist (Cotton) for providing facilities for this project.

\section{References}

Anonymous 2017. ICAR-AICRP (Cotton) Annual Report (2016-2017). ICAR - All India Coordinated Cotton Improvement Project, Coimbatore 641 003, Tamil Nadu.

Asghar Heydari 2015. A comparison between acid seed delinting and fungicide seed treatment in controlling cotton seedling damping-off disease. Intl. J. Agri. Crop. Sci., 8 (4): 573-577.

Bhattiprolu, S.L. and Prasada Rao, M.P. 2009. Estimation of crop losses due to Alternaria leaf spot in cotton. J. Indian Soc. Cotton Improv, 34 (3):151 - 154.

Ebadollah Baniani, Morteza Arabsalmani and Ebrahim Farahani 2015. Effects of seeds treatment with fungicides and insecticides on germination and vigurity, abnormal root producing and protection of cotton seedling. Int. J. Life. Sci. Scienti. Res., 2(5): 519-530.

Eisa (Nawal) A., El-Habbaa, G.M., El-Emery, M. I. and Hassan, S. R. 2007. Efficacy of dressing cotton seeds with fungicides in controlling root pathogens under field conditions. $11^{\text {th }}$ Congress of the Egyptian Phytopathol. Soc., Cairo, 2007.

Hanasi, P. S., 2013. Studies on seed borne diseases of cotton in Northern Karnataka. M.Sc. (Ag) Thesis, University of Agricultural Sciences, Dhawad, India. 
Oktay Erdogan, Yuksel Bolek, M. Erhan Gore 2016. Biological control of seedling diseases by fluorescent Pseudomonas spp. J. Agric. Sci., 22: 398-407.

Sharma, K.K., Singh, U.S., Pankaj Sharma, Ashish Kumar and Lalan Sharma 2015. Seed treatments for sustainable agriculture-A review. J. Appl. \& Nat. Sci.7 (1): $521-539$.

Sheo Raj. 1988. Grading system for cotton diseases, Nagpur. CICR, Tech. Bull., pp.1-7.

Tomer, D. S., Shastry, P. P., Nayak, M. K. and Sikarwar, P. 2012. Effect of seed borne micoflora on cotton seed (JK 4) and their control. J. Cotton Res. Dev., 26(1):105-108.

Wheeler BEJ. 1969. An Introduction to Plant Diseases. John Wiley and Sons Limited, London, p301.

\section{How to cite this article:}

Bhattiprolu, S.L. 2017. Field Efficacy of Seed Dressing Fungicides against Seed Borne Diseases of Cotton. Int.J.Curr.Microbiol.App.Sci. 6(8): 3661-3667. doi: https://doi.org/10.20546/ijcmas.2017.608.443 